\title{
DE LAS CLASES DE GESTIÓN EN LA FAMURP A LA ACCIÓN: EL PLAN ESTRATÉGICO 2017-2021 DE LA SOCIEDAD CIENTÍFICA MÉDICO ESTUDIANTIL PERUANA
}

\author{
FROM MANAGEMENT CLASSES IN FAMURP TO ACTION: \\ THE 2017-2021 STRATEGIC PLAN OF THE PERUVIAN STUDENT MEDICAL SOCIETY \\ Cvetkovic-Vega $A^{1,2,3,4, a}$
}

Se conoce que la participación en Sociedades Científicas de Estudiantes de Medicina (SOCIEMS) permite que los estudiantes de medicina humana adscritos a éstas adquieran competencias principalmente en el ámbito de la investigación científica, sin embargo un aspecto adicional es la adquisición en paralelo de habilidades en gestión'. La mayoría de facultades de medicina de nuestro país incorporan en sus mallas curriculares cursos en gestión en salud dirigidos a estudiantes de medicina de los últimos años; a esta realidad no es ajena la Facultad de Medicina Humana de la Universidad Ricardo Palma (FAMURP), en la cual dicho curso es impartido en el Ciclo $\mathrm{XI}$ del sexto año de la carrera ${ }^{2}$. En dicho curso se brindan los conocimientos, herramientas y metodologías para desarrollar competencias que faciliten la participación en gestión efectiva y eficiente de los establecimientos de salud y servicios basados en la atención primaria de salud³

La capacitación y motivación adquirida fue excelente en el curso impartido por nuestra facultad, es por ello que al finalizar el curso surgio la siguiente pregunta ¿por qué no aplicar lo aprendido para complementar los aspectos organizacionales de las SOCIEMs en nuestro país?, es decir, aplicar lo aprendido a nuestra realidad. Asi mismo después de 4 años de participación activa en SOCEMURP postule a la Presidencia de la Sociedad Científica Médico Estudiantil Peruana (SOCIMEP), siendo elegido por la Asamblea General como Presidente en la ciudad de Trujillo el 20 de diciembre del 2015, asumiendo la honrosa y gran responsabilidad de servir a mis compañeros del país desde el ámbito de la investigación científica en pregrado4.

Una de las necesidades cardinales de la asociación era tener un Plan Estratégico Institucional (PEI) para el período 2017-2021 y resaltar la importancia de éste. Esto suponía una mejora cualitativa fundamental pues permitiría trazar un rumbo al cual todos caminemos en conjunto aportando desde cada una de nuestras SOCIEMs locales, comités y cargos un granito de arena que le permita a SOCIMEP cumplir las metas trazadas para cumplir con su objetivo primordial que es la promoción de la investigación científica en pregrado. Además, esto no solo beneficiaría directamente a la asociación nacional, sino que trazaría un modelo a replicar en las SOCIEMs a nivel local articulando y marcando la dirección en los próximos 5 años de los 40 asociados en 18 regiones de nuestro país que son miembros de la familia SOCIMEP. Luego de 3 Asambleas Generales, 1 en Cusco y 2 en Lima, y contando con la asesoría de la Dra. Fiorella Inga y el Dr. Moisés Apolaya, doctores miembros del Consejo de Asesores de SOCIMEP con experiencia en gestión e investigación, es que el 22 de octubre del presente se aprobó en la VIII Asamblea General Extraordinaria celebrada en Lima el Plan Estratégico Institucional SOCIMEP 2017-2021 cuyos objetivos estratégicos se muestran en la tabla 1. Además, para reforzar el enfoque orientado a la gestión en SOCIEMS y promover la importancia de la gestión en salud en la educación médica, durante el año se realizó un programa que constaba de viajes a 18 regiones del país priorizando las regiones de Piura, Lambayeque, La Libertad, Ancash, Cajamarca, San Martín, Iquitos, Ica, Arequipa, Tacna, Puno, Cusco, Ayacucho, Ucayali, Huánuco, Pasco, Junín, Huacho en Lima y terminando por Lima metropolitana.

\footnotetext{
Facultad de Medicina Humana, Universidad Ricardo Palma, Lima, Perú.

2 Sociedad Científica de Estudiantes de Medicina de la Universidad Ricardo Palma, SOCEMURP.

Sociedad Científica Médico Estudiantil Peruana, SOCIMEP.

${ }^{4}$ Instituto de Investigación en Ciencias Biomédicas, INICIB.

Estudiante de Medicina Humana.
}

Correspondencia: Aleksandar Cvetkovic V. Dirección: Broncino 307, San Borja, Lima, Perú. Celular: +511 964982 676. Correo: aleksandar.famurp@gmail.com

Citar como: Cvetkovic-Vega A. De las clases de gestión en la FAMURP a la acción: El plan estratégico 2017-2021 de la Sociedad Científica Médico Estudiantil Peruana. [Sección para Estudiantes]. Rev. Fac. Med. Hum. 2017;17(1):105-107. DOI 10.25176/RFMH.v17.n1.757 
Sin los conocimientos del curso de gestión en salud, ninguno de estos logros ni políticas desarrolladas hubieran sido posibles. Siendo la Medicina una carrera que permite desarrollarse en muchos ámbitos siguiendo la premisa de aliviar al doliente y salvar vidas, entendí que uno de éstos es la gestión en salud a través de la cátedra que recibí en nuestra FAMURP. El médico gestor en salud trabaja de la mano con el equipo de salud facilitando su trabajo y actuando, muchas veces tras el telón, para que sus colegas que se dedican al ámbito asistencial puedan cumplir su labor siendo así coparticipes en el proceso de salvar vidas. Un mensaje central del curso fue que el mejor administrador de un hospital o un sistema de salud no es un administrador puro: lo es un médico y ese criterio que caracteriza por ser científico y humanista, lo cual es producto de una formación médica, la investigación que genera evidencia para la toma de decisiones y del contacto con el paciente y el sistema de salud.

\section{AGRADECIMIENTOS}

Estando ya en la fase final de mi gestion agradezco de manera especial a la Dra. María del Socorro Alatrista Gutíerrez Vda. De Bambarén, profesora del curso de Gestión en Salud y actual Decana de nuestra Facultad de Medicina Humana, por los conocimientos brindados en sus clases y la motivación que despertó en los temas de la gestión en salud. Todo lo aprendido en aquellas clases teóricas y prácticas de los lunes y miércoles por la tarde han sido puestos en práctica este año. También, quiero agradecer a mis madres Rosa y Diana, pues sin el apoyo incondicional de ellas en todos los aspectos, hubiera sido realmente imposible cumplir con esta tarea y al equipo de 13 líderes estudiantiles miembros de mi Consejo Directivo con los que asumimos este reto para el 2016.

\section{OBJETIVO}

\section{ESTRATÉGICO}

\section{DESCRIPCIÓN}

OE1

Desarrollar programas de promoción de la investigación en salud ya través de pasantías acordes a las prioridades nacionales y regionales con instituciones públicas y privadas.

OE2

Fomentar y fortalecer la publicación en revistas científicas indizadas a nivel nacional e internacional con autoría de estudiantes de medicina.

OE3

Generar y fomentar líneas de investigación científica en el pre-grado entre los asociados de SOCIMEP y aliados estratégicos.

OE4

Diseñar políticas que optimicen los aspectos organizacionales de SOCIMEP en ámbitos de gestión, contabilidad y fortalecimiento del acervo cultural institucional.

OE5

Participar activamente en la promoción de investigación en pregrado en las facultades de los asociados a SOCIMEP.

OE6

Fomentar y promover la ética científica en la elaboración y ejecución de protocolos y en la publicación de investigaciones científicas en pre-grado.

OE7

Impulsar la red de miembros y ex miembros de SOCIMEP enfocada en asesoramiento metodológico en investigación y temas de epidemiología, gestión y salud pública.

OE8

Procurar que las universidades e instituciones públicas y privadas reconozcan al estudiante de medicina que desarrolla investigación durante el pre-grado.

Tabla 1. Objetivos Estratégicos del PEI SOCIMEP 2017-20215. 
Financiamiento: Autofinanciado.

Conflicto de interés: El autor declara no tener conflicto de interés en la publicación de este artículo.

Recibido: 25 de febrero del 2017

Aprobado: 14 de marzo del 2017

\section{REFERENCIAS BIBLIOGRÁFICAS}

1. Mondragón-Cardona Á, Jiménez-Canizales CE, Alzate-Carvajal V. Oportunidades y desarrollo en las sociedades científicas estudiantiles. Cienc E Investig Medico Estud Latinoam [Internet]. 2013 [cited 2014 Aug 5];17(1). Available from: http://www.cimel.felsocem.net/index.php/ CIMEL/article/viewArticle/231

2. Facultad de Medicina Humana de la Universidad Ricardo Palma. Malla Curricular [Internet]. 2014 [cited 2016 Oct 19]. Available from: http:// www.urp.edu.pe/pdf/medicina/estructuracurricular.pdf

3. Facultad de Medicina Humana de la Universidad Ricardo Palma. Sillabus del Curso de Gestión en Salud [Internet]. 2015 [cited 2016 Oct 19]. Available from: http://www.urp.edu.pe/pdf/medicina/silabo-2016.pdf
4. Cvetkovic-Vega A, Mejía CR, Patiño-Calla Karina, Diana Llacta-Aparicio, Cesar Espinoza-Chiong, Mariela Vargas, et al. UN MIEMBRO PROACTIVO DE LA INVESTIGACION.pdf. Rev Fac Med Humana. 2016 de abril del;16(1):62-3.

5. Sociedad Científica Médico Estudiantil Peruana SOCIMEP. Plan Estratégico Institucional SOCIMEP 2017-2021 [Internet]. 2016 [cited 2010 Oct 19]. Available from: http://www.socimep.net/acerca

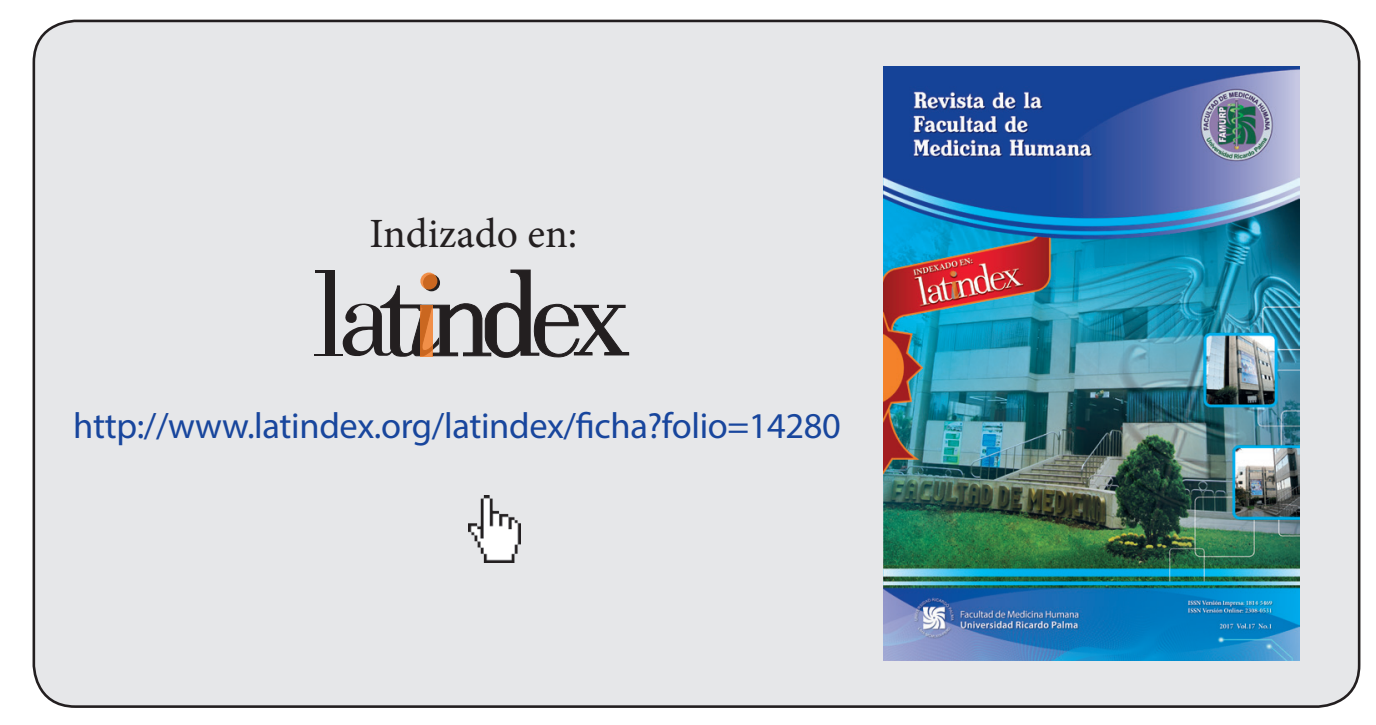

